\title{
REVERSE MICROEMULSION OF IGEPAL CO-720 SYSTEM AS MICROREACTOR FOR CdS SYNTHESIS
}

\author{
Fitria Rahmawati ${ }^{a^{*}}$, Indah Rizki Fitriani ${ }^{\mathrm{a}}$, Abu Masykur ${ }^{\mathrm{b}}$ \\ ${ }^{a}$ Research Group of Solid State Chemistry \& Catalysis, FMIPA, Universitas SebelasMaret, \\ Jl. Ir. Sutami 36 A, Kentingan, Surakarta 57126 telp. (0271) 663375 \\ ${ }^{\mathrm{b}}$ Research Group of Analytical Chemistry, FMIPA, Universitas SebelasMaret, Jl. Ir. \\ Sutami 36 A, Kentingan, Surakarta 57126 telp. (0271)663375 \\ *email: fitria@mipa.uns.ac.id
}

Recieved 18 February 2014, Accepted 18 July 2014, Published 01 September 2014

\begin{abstract}
A Research on CdS synthesis in reverse microemulsion of Igepal CO-720 system has been conducted at various weight ratio of water to surfactant. Igepal CO-720 naturally forms oil in water $(\mathrm{o} / \mathrm{w})$ emulsion type due to its high HLB (Hydrophilic-Lipophilic Balance) value. Therefore, in this research the Igepal CO-720 system was inversed into water in oil (w/o) system before it was used as microreactor for $\mathrm{CdS}$ synthesis. As comparison, a system of AOT (Aerosol OT; sodium bis(2-ethylhexyl) sulfosuccinate) which is naturally w/o system was also used as microreactor for CdS synthesis. The prepared $\mathrm{CdS}$ was analyzed by X-ray diffraction for crystal identification, scanning electron microscope for morphological analysis, $\mathrm{UV}-\mathrm{V}$ is for absorption edge determination and photoelectrochemical testing for photoactivity. The results show that the Igepal CO720 system can be inverted into w/o system and can be used as microreactor for $\mathrm{CdS}$ synthesis. The prepared CdS is in nanosize with the average diameter of $2.517 \pm 0.014 \mathrm{~nm}$ and the average gap energy of $3.805 \pm 0.178 \mathrm{eV}$. The prepared CdS in Igepal CO-720 system has less regular form in comparison with morphology of the prepared CdS in AOT system. As the $\omega$ values decreases the particle diameter decreases, the gap energy increases and the \% IPCE increases. It indicates that high surfactant concentration allows small size micelles formation and produced smaller CdS particle that has high surface area and therefore provide higher photocatalytic activity which was indicated by high value of its $\%$ IPCE.
\end{abstract}

Key words: CdS nanoparticle, Igepal CO-720, microemulsion

\section{INTRODUCTION}

Microemulsion is a system that consists of water and oily components which are undissolved each other. However, both components can form single phase by surfactant addition. The surfactant molecules plays significant role due to their capability to reduce the interface tension between water and oil. Recently, the microemulsion has been applied in many applications such as in food industries, pharmaceutical, cosmetics, agrochemistry, in dyeing process and also in nanoparticle synthesis (Solans and Kuneida, 1997). The growing interest in technological applications of nanoparticle such as for solar cells, light 
emitting diode, medical diagnostics (Nie et al., 2007; Rhyner et al., 2006 and Santra et al., 2004) and photocatalysis (Serpone and Khairutdinov 1997; Zou et al., 2005; Zhang et al., 2003), allows the high rate development on synthesis method in order to produce micro- or nano-size semiconductor. Some researchers found that the optical, optoelectronic and magnetic properties of such materials are depending on the size according to the size quantization effect (Henglein, 1989).

Igepal CO-720 is a non ionic surfactant with HLB number 14.2. Therefore this surfactant tends to form oil in water $(\mathrm{o} / \mathrm{w})$ emulsion. It is known that based on its continuous phase, the micro emulsion system can be classified into two types, i.e. oil in water $(\mathrm{o} / \mathrm{w})$ and water in oil (w/o) system. Those types are related to the surfactant used in micro emulsion formation, especially related to the HLB (Hydrophile-Lipophile Balance) of the surfactant. Surfactant with low HLB number will tend to produce w/o emulsion due to its high solubility in oily solvent and the surfactants with high HLB number will tend to produce o/w emulsion due to its high solubility in water solvent (Moroi, 1988). Nonionic surfactant is more stable relative to ionic surfactant and it is more inexpensive than ionic surfactant. However, in nanoparticle synthesis, w/o microemulsion is required. Therefore, an o/w system which is actually can be produced by inexpensive surfactant must be transformed into w/o emulsion before being used in nanoparticle synthesis. Some researchers usually used AOT (bis-2 (ethylhexyl) sodium sulfosuccinate), a low HLB number surfactant, in microemulsion formation for particle synthesis. Phase transformation of $\mathrm{o} / \mathrm{w}$ emulsion to w/o emulsion can be carried out by the addition of electrolyte and the addition of the organic phase. The phase transformation process can be detected by the change of conductivity of the system (Bourrel and Schechter, 1988). Mc Coy (2002) studied phase inversion of sun seed oil emulsion in water which has been formed by using Igepal CO-520 (HLB 10) and Igepal-720 (HLB 14.2) as surfactants. The phase inversion was done by the addition of oily phase (nonpolar phase) gradually. Meanwhile the inversion point was determined by conductivity measurement.

There are many advantages on nanoparticle synthesis by microemulsion method, i.e. the size and form of particles can be designed by controlling the inner core of the micelles, a surfactant aggregates that formed in microemulsion; this method does not require any specific equipments and also no requirement on high temperature and/or high pressure condition (Hefnawy, 2012). This method also can yield particles nearly monodisperse in size (Tan et al., 2011). Chandler, Bigham and Coffer (1993) prepared $\mathrm{CdS}, \mathrm{PbS}$ and $\mathrm{ZnS}$ particles by microemulsion of water/AOT/n-heptane system. It was founded that the increasing of water to surfactant ratio, $\omega$, produced UV Vis spectrum shifting. The ratio, $\omega=10$, produced red shifting to $450 \mathrm{~nm}$, in relative to $\omega=5$. Therefore 
by designing the $\omega$ value, the photocatalyst particles such as $\mathrm{CdS}$ can be engineered to be more active under visible light area, then the photocatalytic process can be proceed effectively and more economically under sunlight irradiation. Simmons et al. (2002) also synthesized CdS quantum dots from AOT water in oil microemulsion system and found that the change in particle morphology is directly related to the shape of the reversed micelle in which it was synthesized. It indicates the role of surfactant templating.

This paper discusses the synthesis of CdS particles in microemulsion system of water/igepal-720/n-butanol $/ n$-hexane. This research investigated the effect of Igepal CO720 concentration to the properties of synthesized $\mathrm{CdS}$, including the cluster size, photoactive property, crystallinity and crystal system. In order to investigate capability of the synthesized CdS as a photocatalytic agent, a photoelectrochemical testing was carried out. The principal is light radiation with suitable energy can excite electrons from valence bands to conduction bands producing hole $\left(h^{+}\right)$in valence band and electrons $\left(\mathrm{e}^{-}\right)$in conduction. The excited electrons flowed to the external circuit and recorded by a microampermeter. As a comparison, a microemulsion system of water/ AOT/ $n$-butanol $/ n$ hexane also was prepared to be used for $\mathrm{CdS}$ particles. The AOT microemulsion system naturally forms water in oil microemulsion, therefore synthesize can be proceed without inversion steps.

\section{EXPERIMENT}

All chemicals used in this research are in pro analysis grade. Igepal CO-720 and AOT surfactant were procured from Aldrich. The precursors of $\mathrm{CdS}$ are $\mathrm{CdNO}_{3}$ (E-Merck) and $\mathrm{Na}_{2} \mathrm{~S}$ (E-Merck). Meanwhile the nonpolar phase is n-hexane (E-Merck) and the additive is $n$-butanol (E-Merck). Chemicals for photoelectrochemistry testing are TEAI (Tetra Ethyl Amonium Iodide) (Aldrich), $\mathrm{I}_{2}$ (E-Merck) and Acetonitril (E-Merck).

The inversion point was determined from the conductivity curve as function of $n$ hexane volume. Droplet size was determined by sedimentation technique. XRD analysis (X-rays Diffractometer Shimadzu XRD-6000) was used to investigate the crystallinity and crystal system of CdS. Meanwhile, the surface morphology was analyzed from SEM (JEOL JSM-5310 LV) images. The absorption edge which was determined from solid phase UV Vis spectrum was used to calculate the band gap energy of the synthesized CdS. Solid phase UV-Vis spectrum was recorded by coating the CdS solution on a glass preparate that produced very thin layer of $\mathrm{CdS}$ after being dried under room temperature. The coated glass preparate was then being analyzed by UV-Vis spectrophotometer (Double Beam Shimadzu UV-1601 PC) with a blank preparate as control. 
The inversion process also was investigated through the change of images recorded by Microscope Optic equipped with Camera (Nikon Eclipse E 400) and through the change of the conductivity. The conductivity was measured by Conductometer Jenway CE 4071.

\section{Phase inversion}

A $3 \mathrm{~mL}$ Igepal Co-720 was dissolved into the mixture of $10 \mathrm{~mL}$ of aquades, $10 \mathrm{~mL}$ of $\mathrm{n}$-hexane and $5 \mathrm{~mL}$-butanol. The mixture was stirred by an impeller motor (KikaLabortechnique) with $300 \mathrm{rpm}$ of rate for 2 minutes. The conductivity was then being measured by ConductometerJenway CE 4071. Then, $5 \mathrm{~mL}$ of $\mathrm{n}$-hexane was added into the mixture and was stirred for 2 minutes before conductivity measurement. This procedure was repeated until the conductivity of the solution was constant. During inversion process, the droplet forms of emulsion was recorded by taken the images of a little drop of emulsion under optical microscope equipped with camera.

\section{The CdS synthesis}

A $1 \mathrm{~mL}$ of $\mathrm{Na}_{2} \mathrm{~S}(1 \mathrm{M})$ solution was poured into $20 \mathrm{~mL}$ of the inversedmicroemulsion and then being stirred for 4 minute at $300 \mathrm{rpm} .1 \mathrm{~mL}$ of $1 \mathrm{M} \mathrm{Cd}\left(\mathrm{NO}_{3}\right)_{2}$ was added during stirring process. After stirring was stopped, CdS deposit was formed in the bottom of the glass. The deposit was then being filtered with Buchner filtration (Buchner KnF Neuberger D-79112 Fresburg). The deposit then was heated in oven at $120{ }^{\circ} \mathrm{C}$ for 30 minutes and continued with crystal analysis, morphological analysis and photoelectrochemical test.

\section{CdS synthesis in microemulsion system of water/AOT/n-butanol/n-hexane}

A $1.88 \mathrm{~g}$ of AOT powder was poured into the mixture of $10 \mathrm{~mL} n$-hexane, $5 \mathrm{~mL} n$ butanol and being stirred at $300 \mathrm{rpm}$ for 2 minute. A $5 \mathrm{~mL} \mathrm{n}$-hexane was added while the mixture is stirred. The $n$-hexane addition was repeated until the total volume added was 50 $\mathrm{mL}$. Then $10 \mathrm{~mL}$ water was added into the mixture and was stirred to form w/o emulsion. The procedure was repeated with $2.504 \mathrm{~g}$ of AOT and also for $3.129 \mathrm{~g}$ of AOT. The AOT weight is comparable to ratio of water to surfactant $121.667,90.671$ and 72.564 .

$1 \mathrm{~mL}$ of $\mathrm{Na}_{2} \mathrm{~S}(1 \mathrm{M})$ solution was added into $20 \mathrm{~mL}$ of the prepared microemulsion and followed by stirring at $300 \mathrm{rpm}$ for 2 minutes. Then, $1 \mathrm{~mL}$ of $\mathrm{Cd}\left(\mathrm{NO}_{3}\right)_{2}(1 \mathrm{M})$ solution was added while the mixture was kept under stirring. The deposit was then filtered with Buchner filtration and the deposit was heated at $120{ }^{\circ} \mathrm{C}$ for 30 minutes. The synthesized$\mathrm{CdS}$ then was ready to be characterized.

$\mathrm{CdS}$ was synthesized at various concentrations of Igepal CO-720 and AOT as listed in Table 1. 
Table1. Various surfactant concentrations that were used in CdS synthesis and the $\omega$ values. $\omega$ is water to surfactant ratio.

\begin{tabular}{cccc}
\hline Igepal CO-720 $(\mathrm{mL})$ & [Igepal CO-720] $\mathrm{M}=[\mathrm{AOT}] \mathrm{M}$ & AOT $(\mathrm{g})$ & $\omega$ \\
\hline 3 & 0.062 & 1.878 & 121.667 \\
4 & 0.082 & 2.503 & 90.671 \\
5 & 0.101 & 3.129 & 72.564 \\
\hline
\end{tabular}

\section{Sedimentation technique for droplet size estimation}

In order to estimate the droplet size of microemulsion, $5 \mathrm{~mL}$ of the prepared microemulsion was poured into a cuvet then being centrifuged (Kokusan H-107 $(50$ Hz, $4000 \mathrm{rpm})$ ) at $2500 \mathrm{rpm}$ for 30 minutes. The microemulsion became separated into two layers. The droplet size was calculated with equation (1). Meanwhile, the microemulsion and $n$-hexane density were determined by using picnometer and the viscosity was determined by Ostwald method.

$$
\mathrm{V}=\frac{\mathrm{dX}}{\mathrm{dt}}=\frac{2 \operatorname{gr}^{2}\left(\rho-\rho_{\mathrm{m}}\right)}{9 \eta}
$$

$\mathrm{V}$ is deposition velocity which is the velocity of sedimentation $(\mathrm{m} / \mathrm{s})$. The velocity is a function of $\mathrm{dX}$, a different between $\mathrm{X} 2$ and $\mathrm{X} 1$, in which $\mathrm{X} 1$ is the distance of second layer of microemulsion before centrifugation (m) and $\mathrm{X} 2$ is the distance of second layer of microemulsion after centrifugation $(\mathrm{m}), \mathrm{g}$ is the acceleration of gravity $\left(\mathrm{m} / \mathrm{s}^{2}\right), \mathrm{r}$ is radius of droplet, $\rho$ is the density of microemulsion, $\rho_{\mathrm{m}}$ is the density of medium and $\eta$ is viscosity of medium.

The energy gap of CdS particle is calculated with equation (2). The absorpsion edge was determined by UV Vis spectrophotometer measurement of thin layer CdS on glass preparate with a blank glass as a control.

$$
\mathrm{E}_{\mathrm{g}}=\mathrm{h} \frac{\mathrm{c}}{\lambda_{\mathrm{g}}}
$$

$\mathrm{E}_{\mathrm{g}}$ is gap energy of $\mathrm{CdS}(\mathrm{eV})$, $\mathrm{h}$ is Plack constant $\left(4.14 \times 10^{-15} \mathrm{eV} . \mathrm{s}\right)$, c is the velocity of light $(\mathrm{m} / \mathrm{s})$ and $\lambda_{\mathrm{g}}$ is the absorption edge $(\mathrm{m})$.

\section{Photoelectrochemical testing}

The photoelectrochemical test aimed to investigate the efficiency of conversion of photon to electrical current which is defined as \% IPCE (\% Induced Photon to Current Efficiency). The scheme of measurement is depicted in Figure 1. 


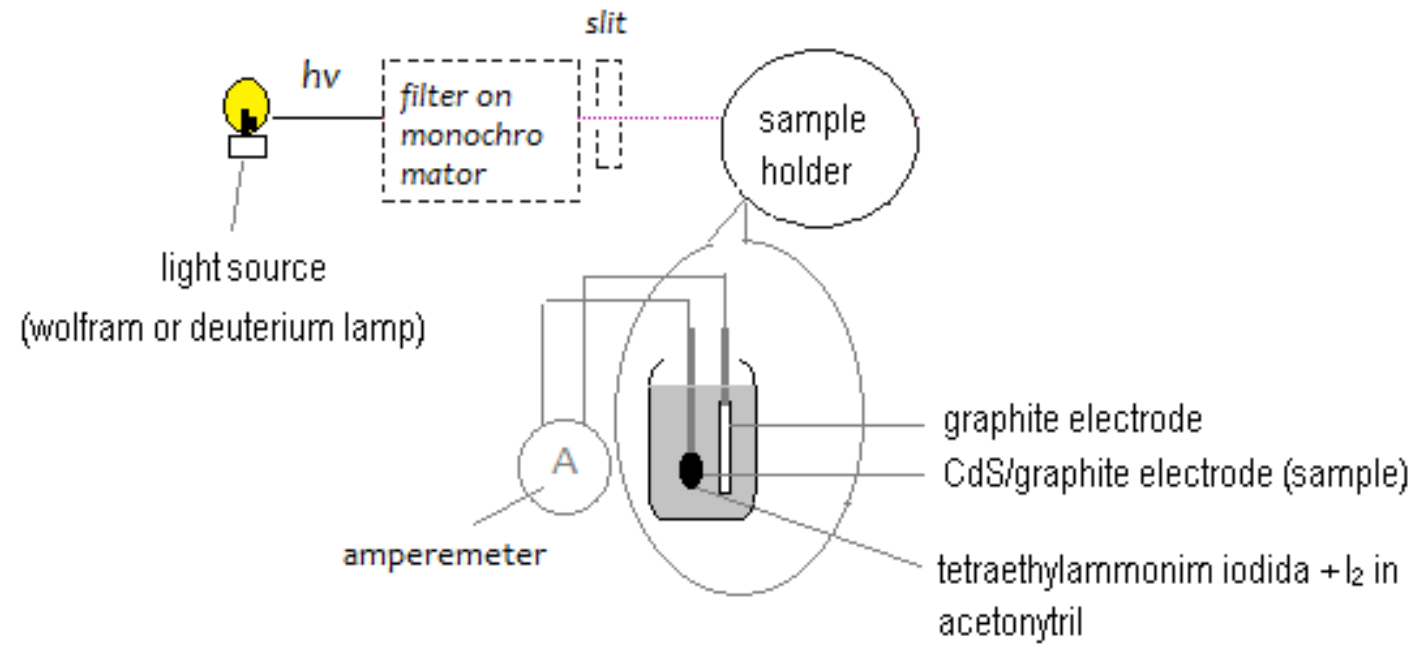

Figure 1. The scheme of photoelectrochemical testing.

A graphite substrate was prepared from graphite powder which was pressed under 400 bars in a mold with geometry of $5.3 \times 2.6 \times 0.5 \mathrm{~cm}$. Meanwhile, 0.05 gram of CdS powder was dissolved in Triton-X (e-merck) solution. The solution was then coated on the graphite substrate and followed by heating at $80{ }^{\circ} \mathrm{C}$ for 30 minutes. The CdS/graphite was

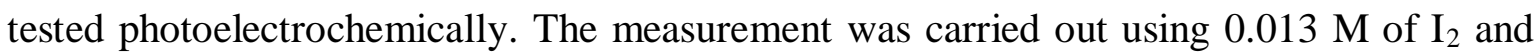
$0.157 \mathrm{M}$ of $\left(\mathrm{C}_{2} \mathrm{H}_{5}\right)_{4} \mathrm{NI}$ solution in $20 \mathrm{~mL}$ of acetonitril. The absorbance was recorded at wavelength of $300-800 \mathrm{~nm}$. The current was recorded and then \%IPCE was calculated using equation (3).

$$
\% \text { IPCE }=\frac{\mathrm{I}_{\mathrm{sc}}\left(\mathrm{A} / \mathrm{cm}^{2}\right)}{\mathrm{I}_{\mathrm{inc}}\left(\mathrm{W} / \mathrm{cm}^{2}\right)} \times \frac{1240}{\lambda(\mathrm{nm})} \times 100 \%
$$

$I_{s c}$ is the current measured in every wavelength, $I_{\text {inc }}$ is the power of light that induces the $\mathrm{CdS}$ surface and $\lambda$ is the wavelength of incident light.

\section{DISCUSSION}

The phase inversion of microemulsion system of water/Igepal CO-720/n-butanol/nhexane which was carried out by $n$-hexane addition gradually produced the change in conductivity as depicted in Figure 2. It is found that the inversion points of microemulsion with various Igepal volume are at $30 \mathrm{~mL}$ of hexane addition and become stabilized after 35 $\mathrm{mL}$ of $n$ - hexane addition. 


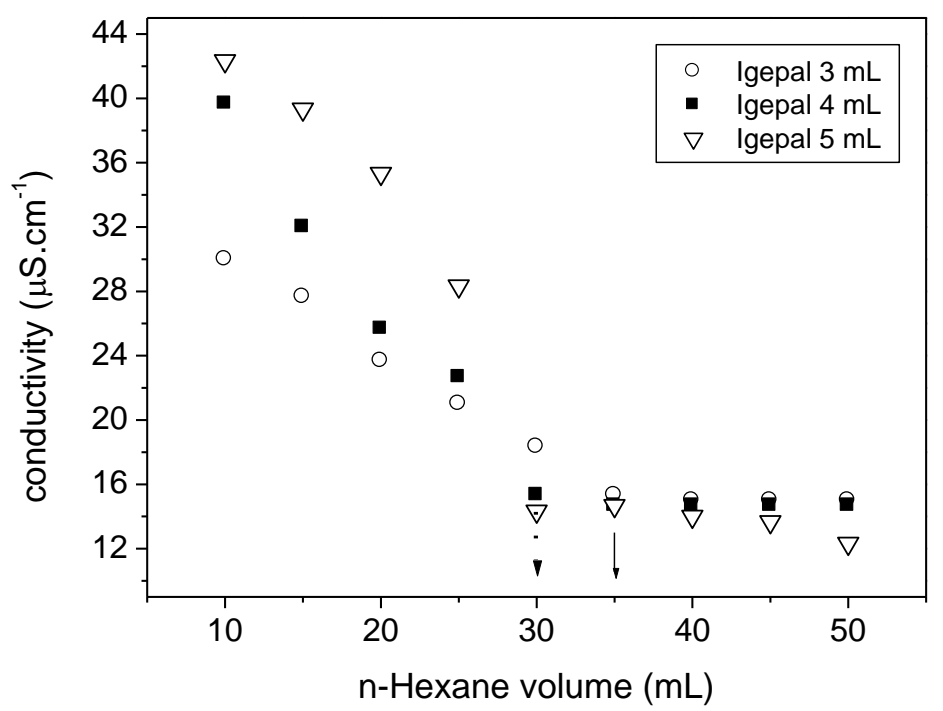

Figure 2. Conductivity of microemulsion system of water/Igepal CO-720/n-butanol/ $n$ hexane as function of $n$-hexane addition.

In microemulsion, water molecules as polar continuous phase interact with the head of surfactant Igepal CO-720 which is also polar group. The polar part of surfactant molecules bearing counter ions and therefore the microemulsion has high conductivity. Meanwhile, the tail group which is non polar interacts with $n$-hexane molecules and forming internal phase of micelle. A micelle is aggregate of some surfactant molecules. In this system the core of a micelle is filled with hexane and the microemulsion type is oil in water or $\mathrm{o} / \mathrm{w}$ emulsion. The addition of $n$-hexane increase the contact area with $n$-hexane molecules, meanwhile the contact area with water molecules decrease and finally $n$-hexane become continuous phase with the tail of surfactant are oriented outside to $n$-hexane as the new continuous phase. This allows decreasing of conductivity due to $n$-hexane as nonpolar molecules act as good medium for charge migration. The inversion process is completed when the conductivity become constant. In this research, the inversion point is found at $30 \mathrm{~mL}$ addition of $\mathrm{n}$-hexane as it is described in Figure 2.

The inversion process also can be detected through morphological changes found in the microemulsion images which were recorded by the optical microscope at $100 \mathrm{x}$ magnification. Figure 3 shows that the droplet size decreased as the volume of $n$-hexane increases. The color of internal phase or core of droplet transformed from white into black, meanwhile the continuous phase transformed from black into white. 

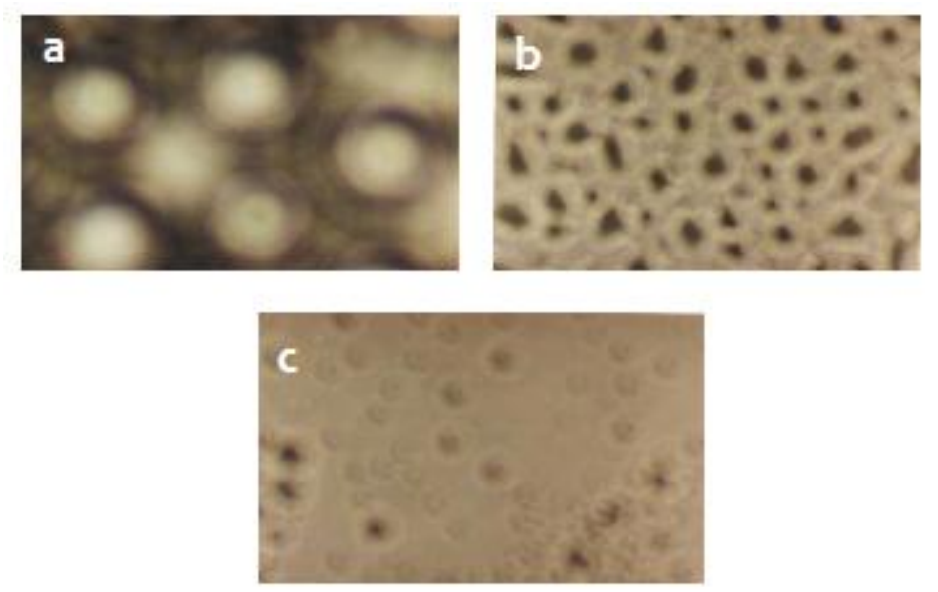

Figure 3. The images of transformation of droplet size and droplet form during inversion process. (a) droplet at $15 \mathrm{~mL}$ of $n$-hexane addition, (b) droplet at $30 \mathrm{~mL}$ of $n$-hexane addition and (c) droplet at $35 \mathrm{~mL}$ of $n$-hexane addition.

The droplet form of water/AOT/n-butanol $/ n$-hexane is depicted in Figure 4 . This microemulsion system is a w/o emulsion type since beginning due to AOT is a nonionic surfactant with low HLB value.

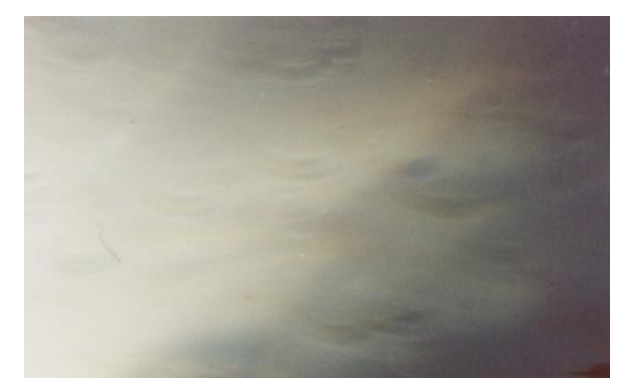

Figure 4. The image of microemulsion of water/AOT $/ n$-butanol $/ n$-hexane system.

Sedimentation technique was used to estimate the radius of droplet. The technique measured the distance between layers which were formed by centrifugation and continued with calculation using equation (1). The radius of droplets is listed in Table 2. It is known that droplets formed in water/AOT/n-butanol $/ n$-hexane system have smaller size than the droplets formed in water/Igepal CO-720/n-butanol/n-hexane system. It is also known that the droplet size decreases as the ratio of water to surfactant decreases and comparable with the study on microemulsion droplet size by mc Coy (2002) who found that the increasing of surfactant molecules in a micellar solution allows decreasing of space between head group of surfactants in the interface area of surfactant and water, then the droplet size is become smaller. Solanksi et al. (2010) also found that silver particles that were synthesized in water/AOT/cyclohexane microemulsion system were smaller and narrower in the size distribution at lower water content than that obtained at high $\omega$ value. 
Table 2. The radius of droplet as estimated by sedimentation technique.

\begin{tabular}{lrr}
\hline surfactant & $\omega$ microemulsion & $\mathrm{r}(\mu \mathrm{m})$ \\
\hline Igepal C0-720 & 121.667 & 3.10 \\
& 90.671 & 2.99 \\
AOT & 72.564 & 2.54 \\
& 121.667 & 1.16 \\
& 90.671 & 0.79 \\
& 72.564 & 0.35 \\
\hline
\end{tabular}

The reaction of $\mathrm{Cd}\left(\mathrm{NO}_{3}\right)_{2}$ solution and of $\mathrm{Na}_{2} \mathrm{~S}$ solution in the inversed microemulsion is described in equation (4). The reaction produced yellow sediment which was collected from the bottom of reaction glass.

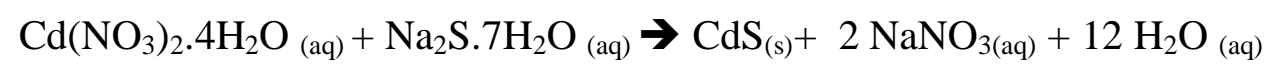

During reaction the $\mathrm{Cd}^{2+}$ ions migrate into the internal phase of micelles which was filled by $\mathrm{S}^{2-}$ ions (Figure 5). The reaction between $\mathrm{Cd}^{2+}$ and $\mathrm{S}^{2-}$ occured and produced $\mathrm{CdS}$ particles with size that matched with the size of internal phase or core micelle. Therefore, in this process, the internal phase served as microreactor for $\mathrm{CdS}$ particles formation. Weight of the synthesized CdS that produced at different ratio of water to surfactant, $\omega$, are listed in Table 3.

Tabel 3. Weight of the synthesized CdS at different water to surfactant ratio, $\omega$

\begin{tabular}{lrr}
\hline surfactant & $\omega$ & Weight of CdS $(\mathrm{g})$ \\
\hline Igepal CO-720 & 121.667 & 0.292 \\
& 90.671 & 0.438 \\
AOT & 72.564 & 0.371 \\
& 121.667 & 0.167 \\
& 90.671 & 0.249 \\
& 72.564 & 0.517 \\
\hline
\end{tabular}




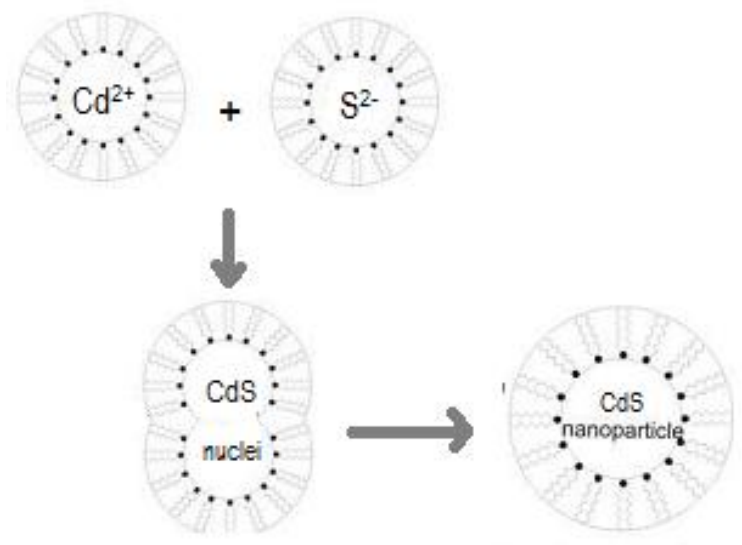

Figure 5. The schematic of CdS formation.

Morphological analysis of the prepared CdS with SEM produced images as depicted in Figure 6 and 7.
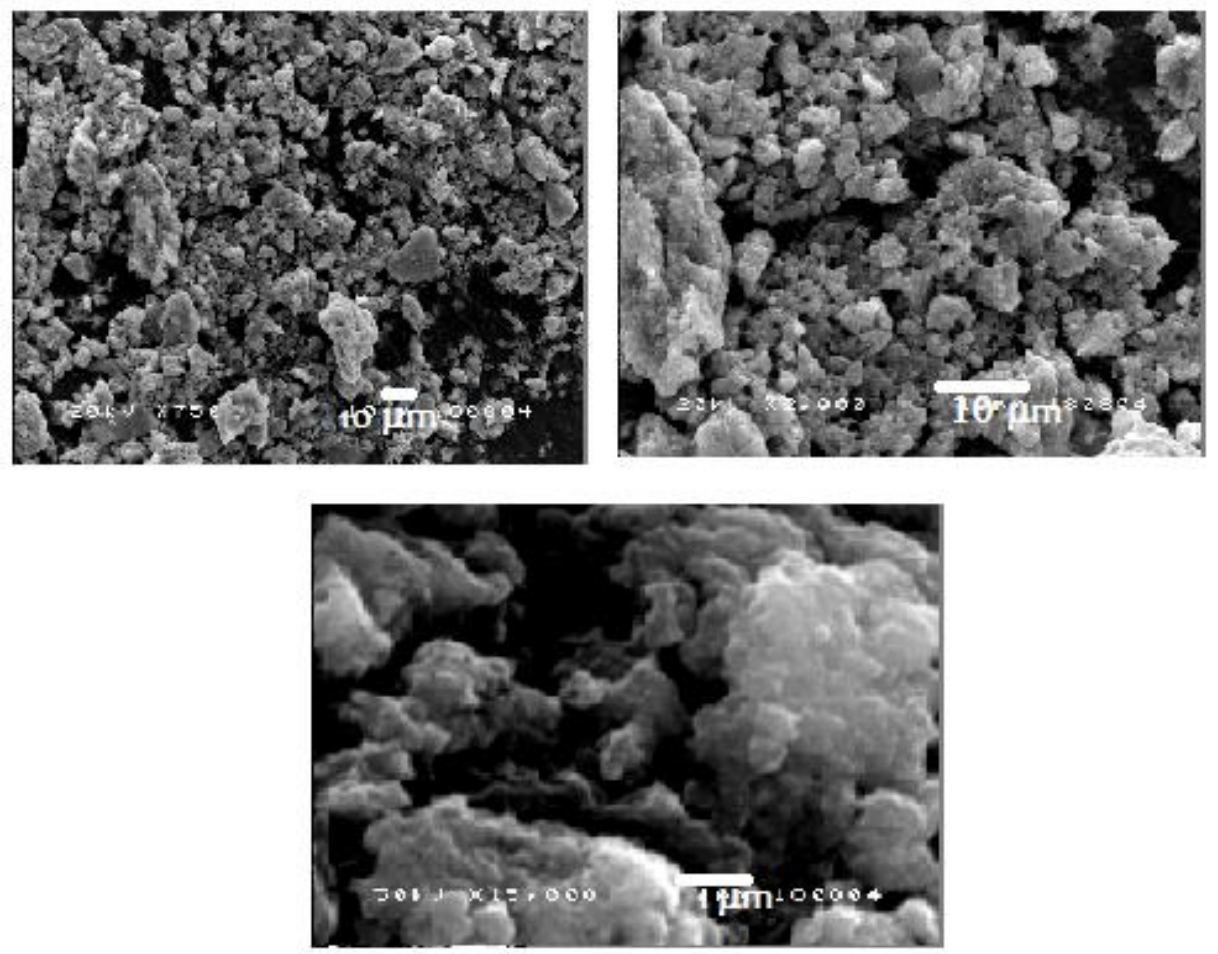

Figure 6. SEM images of CdS which was prepared in water/Igepal CO-720/n-butanol/ nhexane system at different magnification. 

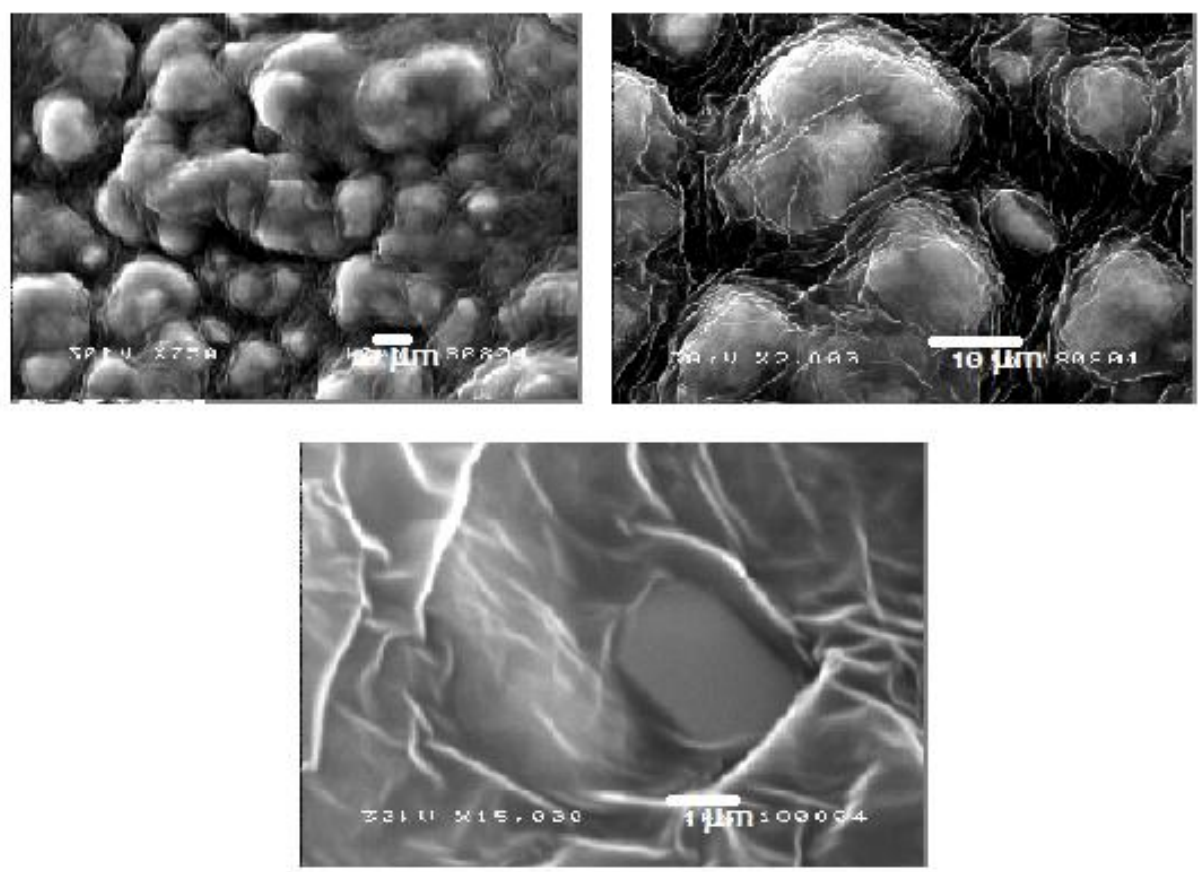

Figure 7. SEM images of the prepared CdS in water/AOT/ $n$-butanol $/ n$-hexane system at different magnification.

Figure 6 and 7 show that the CdS particles produced by microemulsion with AOT surfactant have regular form than the CdS produced by microemulsion with Igepal CO720. However, the CdS whether produced by Igepal CO-720 or AOT agglomerated into larger droplet. This is probably because of surfactant molecules are still embedded and the surfactant molecules keep contacted to water molecules then allows the particles to agglomerate. It indicates that a post treatment is required to get better dispersed CdS. The broaden peaks of X-ray diffraction (Figure 8) indicates that the CdS particles are in small size or even nanosize. However, SEM images confirm that they are agglomerated into larger droplet.

The nanosize of CdS particles was also confirmed by calculation using equation (1). This equation used the absorption edge data which were founded by UV Vis spectrum, as conducted by Nedelkovic et al. (1993). UV Vis spectrophotometer recorded the electronic transition that occurred in $\mathrm{CdS}$ due to light radiation. The electronic spectrums depicted in Figure 9 and 10. 


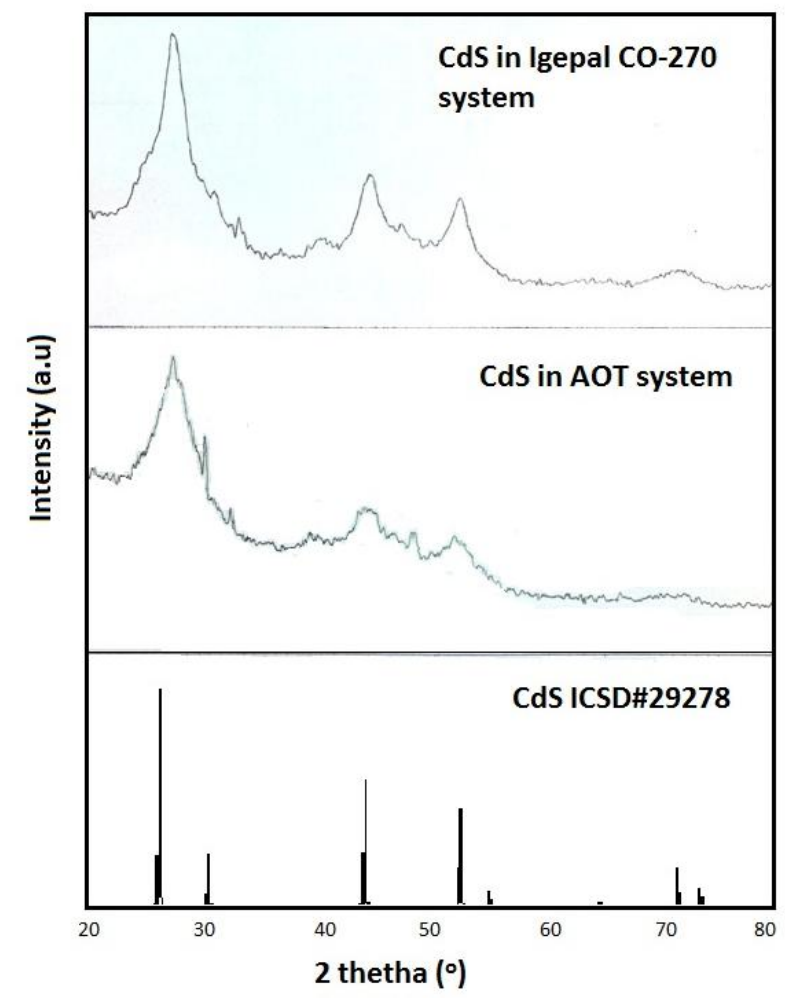

Figure 8. XRD pattern of the prepared CdS.

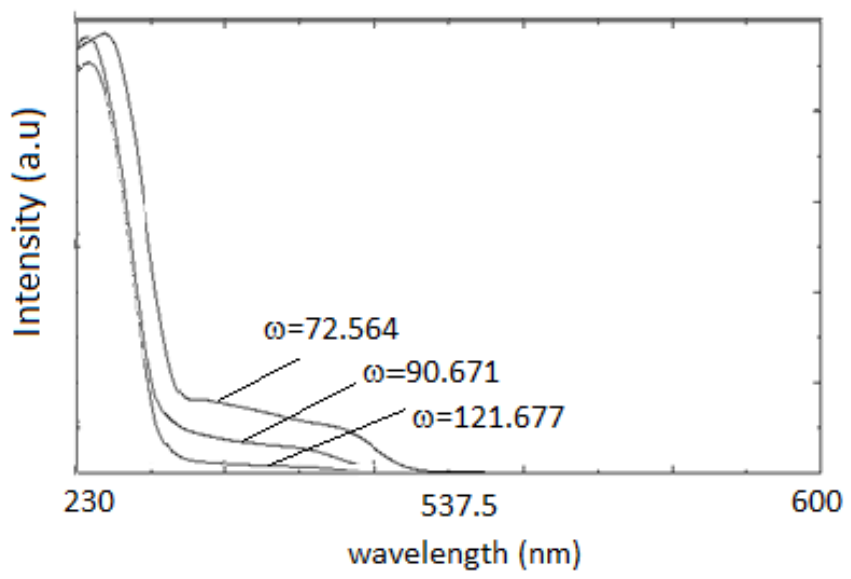

Figure 9. The electronic spectrum of the prepared CdS in water/Igepal Co-720/nbutanol $/ n$-hexane microemulsion at various Igepal CO-720 volumes.

The absorbsion edge, $\lambda_{\mathrm{g}}$, which was determined from its electronic spectrum, was used to calculate the energy gap and diameter of particle by using equation (2). The results are listed in Table 4. 


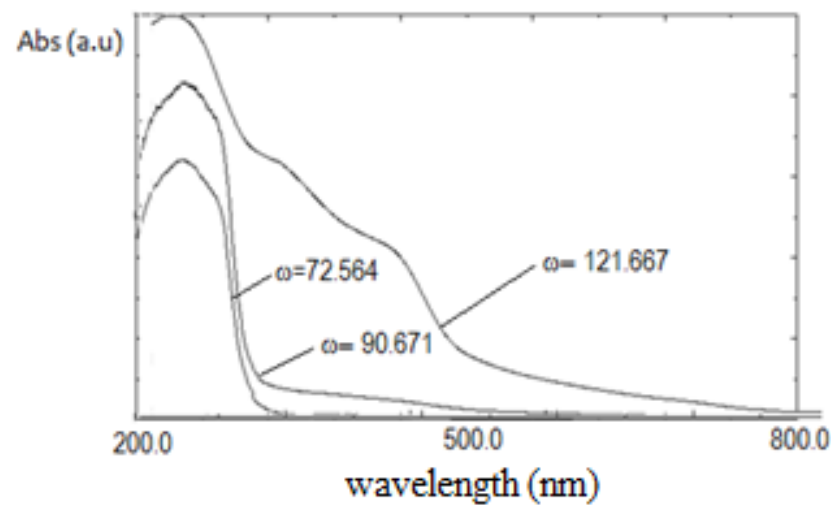

Figure 10. The electronic spectrum of the prepared $\mathrm{CdS}$ in water/AOT $/ n$-butanol $/ n$-hexane microemulsion at various ratio of water to surfactant.

Table 4. The absorption edge, $\lambda_{\mathrm{g}}$, gap energy, $\mathrm{E}_{\mathrm{g}}$ and diameter of $\mathrm{CdS}$ particle at different ratio of water to surfactant.

\begin{tabular}{lrrrr}
\hline \multicolumn{1}{c}{ Surfactant } & \multicolumn{1}{c}{$\omega$} & $\lambda_{\mathrm{g}}(\mathrm{nm})$ & $\mathrm{E}_{\mathrm{g}}(\mathrm{eV})$ & $\begin{array}{c}\text { Diameter of CdS } \\
\text { particle }(\mathrm{nm})\end{array}$ \\
\hline Igepal CO-720 & 121.667 & 335.259 & 3.699 & 2.528 \\
& 90.671 & 334.564 & 3.706 & 2.522 \\
AOT & 72.564 & 309.137 & 4.011 & 2.502 \\
& 121.667 & 405.037 & 3.061 & 3.520 \\
& 90.671 & 305.401 & 4.060 & 2.248 \\
& 72.564 & 303.505 & 4.086 & 2.230 \\
\hline
\end{tabular}

Table 4 shows that the absorption edge, $\lambda_{\mathrm{g}}$, decreases or shifted to hipsochromic and the particle size also decrease as the ratio of water to surfactant, $\omega$, decreases. This results are in agreement with the previous study that was conducted by Solanki et al. (2010). The decreasing of droplet size is related to the decreasing of core size or the size of microreactor as the $\omega$ decrease. The core size has been estimated by sedimentation technique and listed in Table 2.

Theoretically, the smaller particle will have larger surface area and consequently will have high photocatalytic activity. In order to investigate their photocatalytic activity, in this research the prepared $\mathrm{CdS}$ was coated on graphite plate and provide the photoelectrochemical testing at $300 \mathrm{~nm}-800 \mathrm{~nm}$ of light wavelength. The current that was measured is being used to calculate \% Induce photon to current efficiency using equation (3). The results are listed in Table 5.

Table 5 shows that the lower the $\omega$ value, provide higher \%IPCE. This is due to lower $\omega$ value produces smaller particle size that has larger surface are and higher photocatalytic activity than larger CdS particles. In addition, smaller particle size provide larger gap energy and reduces the possibility of electron - hole recombination. This also 
contributes to increase the photocatalytic activity, even though larger gap energy need high energy of UV light to excites electron from valence band to conduction band.

Table 5. The \% IPCE of the prepared CdS at $300 \mathrm{~nm}-800 \mathrm{~nm}$ of light wavelength.

\begin{tabular}{lrrrrrr}
\hline$\lambda(\mathrm{nm})$ & \multicolumn{6}{c}{$\%$ IPCE $\left(\mathrm{x} 10^{-2}\right)$} \\
\cline { 2 - 7 } & \multicolumn{7}{c}{ Igepal CO-720 } & \multicolumn{4}{c}{ AOT } \\
\cline { 2 - 7 } & $\omega=121.677$ & $\omega=90.671$ & $\omega=72.564$ & $\omega=121.677$ & $\omega=90.671$ & $\omega=72.564$ \\
\hline 300 & 4.86 & 5.29 & 5.35 & 4.61 & 4.96 & 5.41 \\
350 & 4.16 & 4.54 & 4.59 & 3.95 & 4.25 & 4.64 \\
400 & 3.65 & 3.77 & 3.83 & 3.27 & 3.72 & 4.06 \\
450 & 3.08 & 3.36 & 3.41 & 2.92 & 3.32 & 3.62 \\
500 & 2.77 & 3.02 & 3.06 & 2.48 & 2.98 & 3.25 \\
550 & 2.51 & 2.60 & 2.65 & 2.25 & 2.57 & 2.82 \\
600 & 2.20 & 2.27 & 2.43 & 2.07 & 2.24 & 2.59 \\
650 & 2.03 & 2.09 & 2.24 & 1.91 & 2.06 & 2.39 \\
700 & 1.88 & 1.94 & 2.08 & 1.77 & 1.92 & 2.11 \\
750 & 1.75 & 1.81 & 1.94 & 1.65 & 1.79 & 1.96 \\
800 & 1.64 & 1.70 & 1.82 & 1.55 & 1.68 & 1.84 \\
\hline
\end{tabular}
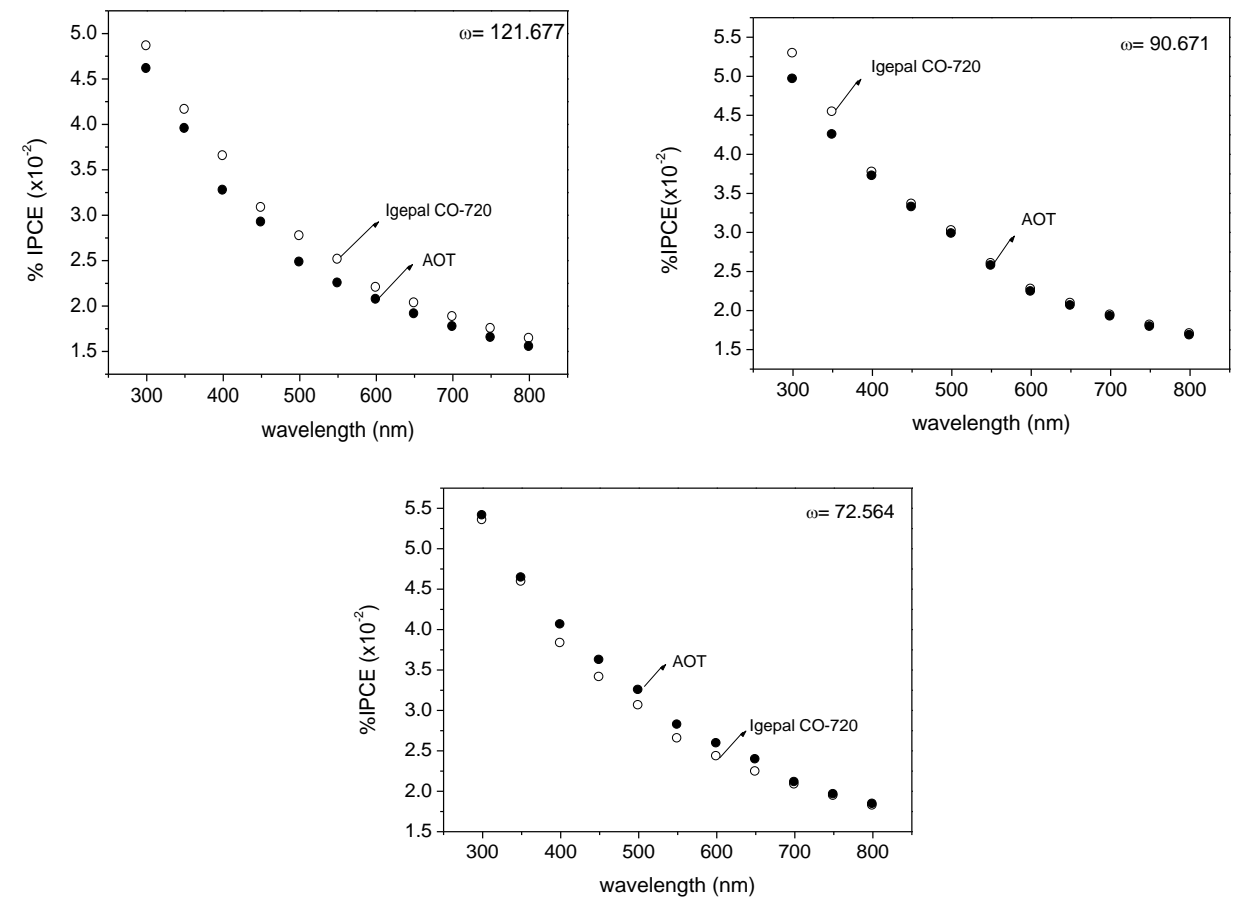

Figure 11. The comparison of \% IPCE of CdS prepared with AOT and Igepal Co-720 microemulsion system at various $\omega$ values.

Even though the surface morphology of CdS that was prepared by Igepal CO-720 microemulsion system is different with the CdS that was prepared by AOT microemulsion system, however their \% IPCE value are almost similar in each $\omega$ values. Figure 10 
provides the comparison of \% IPCE between CdS that was prepared by Igepal CO-720 and AOT microemulsion system.

\section{CONCLUSION}

Microemulsion system of Igepal CO-720 can be inversed to w/o system and used as microreactor for CdS synthesis. The prepared CdS is in nanosize with the average diameter $2.517 \pm 0.014 \mathrm{~nm}$ with the average gap energy is $3.805 \pm 0.178 \mathrm{eV}$. Meanwhile, synthesized CdS particle in AOT system has the average diameter of $2.666 \pm 0.739 \mathrm{~nm}$ and the average energy gap of $3.736 \pm 0.584 \mathrm{eV}$. The morphology of synthesized CdS in Igepal CO-720 system is different with the CdS from AOT system. However, their \% IPCE values are almost similar at various ratio of water to surfactant. As the $\omega$ values decreases the particle diameter decreases, the gap energy increases and the \%IPCE increases. It indicates that high surfactant concentration allows small size micelles formation and produced smaller CdS particle that has high surface area and therefore provide higher photocatalytic activity.

\section{AKNOWLEDGMENTS}

Authors express gratitude to Directorate General of Higher Education (DIKTI), Republic Indonesia for funding this research through Hibah Penelitian Dasar 2005. Thanks to Ernia Agustin for good collaboration during experimental part.

\section{REFERENCES}

Bourrel, M. and Schechter, R.S., 1998, Microemulsions and Related Systems: Formulation, Solvency and Physical Properties, Marcel Dekker, Inc., New York.

Chandler, R.R., Bigham, S.R., and Coffer, J.L., 1993, Spectroscopy Analysis Of Semiconductor Colloids: An Experiment In Materials Science For Advanced Inorganic or Physical Chemistry Laboratory, Journal of Chemical Education, vol.70, no. 1, pp. A7-A10.

El-Hefnawy, M.E., 2012, Water in Olive Oil SurfactantlessMicroemulsions as Medium for CdS Nanoparticles Synthesis, Modern Applied Science, vol. 6, no. 4.

Henglein, A., 1989, Small-particle Research: Physicochemical Properties Of Extremely Small Colloidal Metal and Semiconductor Particles, Chemical Reviews, vol. 89, no. 8, pp. 1861-1873.

Mc Coy, M., 2002, Emulsification of Neara Phase Inversion Condition, http://www.lboro.ac.uk/department/cg/projects/2002/mccoy.

Moroi, Y., 1992, Micelles: Theoritical and Applied Aspects, Plenum Press, New York. 
Nedeljkovic, J.M., Patel, R.C., Kafman, P., Pruden, C.J., and O’Leary, N., 1993, Synthesis and Optical Properties of Quantum Size Metal Sulfide Particles in Aqueous Solutions, Journal of Chemical Education, vol.70, no. 4, pp. 342-345.

Nie, S., Xing, Y., Kim, G. J., and Simons, J. W., 2007, Nanotechnology Applications in Cancer, Annual Review of Biomedical Engineering, vol. 9, pp. 257-288.

Rhyner, M. N., Smith, A. M., Gao, X., Mao, H., Yang, L., and Nie, S., 2006, Quantum Dotsand Multifunctional Nanoparticles: Newcontrast Agents for Tumor Imaging, Nanomedicine, vol. 1, no. 2, pp. 209-217.

Santra, S., Xu, J., Wang, K., and Tan, W., 2004, Luminescent Nanoparticle Probes for Bioimaging, Journal of Nanoscience and Nanotechnology, vol. 4, no. 6, pp. 590599.

Serpone, N. and Khairutdinov, R., 1997, Application of Nanoparticles in ThePhotocatalytic Degradation of Water Pollutants, Studies in Surface Science and Catalysis, vol. 103, pp. 417-444.

Simmons, B.A, Li, S., John, V.T., McPherson, G.L., Bose, A., Zhou, W., and He, J., 2002, Morphology of CdSNanocrystals Synthesized in a Mixed Surfactant System, Nano Letters., vol. 2, no. 4, pp. 263-268.

Solans, C. and Kuneida, H., 1997, Industrial Application of Microemulsions, Marcel Dekker, Inc., New York.

Solanki, J.N. and Murthy, Z.V.P., 2010,Highly monodisperse and sub-nano silver particles synthesisvia microemulsion technique, Colloids and Surfaces A: Physicochemical and Engineering Aspects, vol. 359, pp. 31-38.

Tan, T. T. Y., Liu, S., Zhang, Y., Han, M. Y., and Selvan, S. T., 2011, Microemulsion preparative Methods (Overview), Comprehensive Nanoscience and Technology, vol. 5, pp. 399-441.

Zou, J. J., Chen, C., Liu, C. J., Zhang, Y. P., Han, Y., and Cui, L., 2005, Pt nanoparticles on $\mathrm{TiO} 2$ with novel metal-semiconductor interface as highly efficient photocatalyst, Materials Letters, vol. 59, no. 27, pp. 3437-3440.

Zhang, W., Zhong, Y., and Fan, J., 2003, Preparation, morphology, size quantization effect and photocatalytic properties of CdSQ-nanocrystals, Science China Chemistry B, vol. 46, no. 2, pp.196-206. 